Ebisu Ebisu

Études japonaises Études japonaises

49 | printemps-été 2013

De chose en fait : la question du milieu

\title{
Peut-il exister des terroirs du saké ?
}

Is There Such a Thing as Terroir for Sake?

日本酒にテロワールはあるか

\section{Nicolas Baumert}

\section{CpenEdition}

Journals

Édition électronique

URL : http://journals.openedition.org/ebisu/698

DOI : 10.4000/ebisu.698

ISSN : 2189-1893

Éditeur :

Institut français de recherche sur le Japon (UMIFRE 19 MAEE-CNRS), Maison franco-japonaise

Édition imprimée

Date de publication : 1 avril 2013

Pagination : 5-29

ISSN : $1340-3656$

Référence électronique

Nicolas Baumert, «Peut-il exister des terroirs du saké ? », Ebisu [En ligne], 49 | printemps-été 2013, mis en ligne le 04 avril 2014, consulté le 02 mai 2019. URL : http://journals.openedition.org/ebisu/698 ; DOI : 10.4000/ebisu.698 


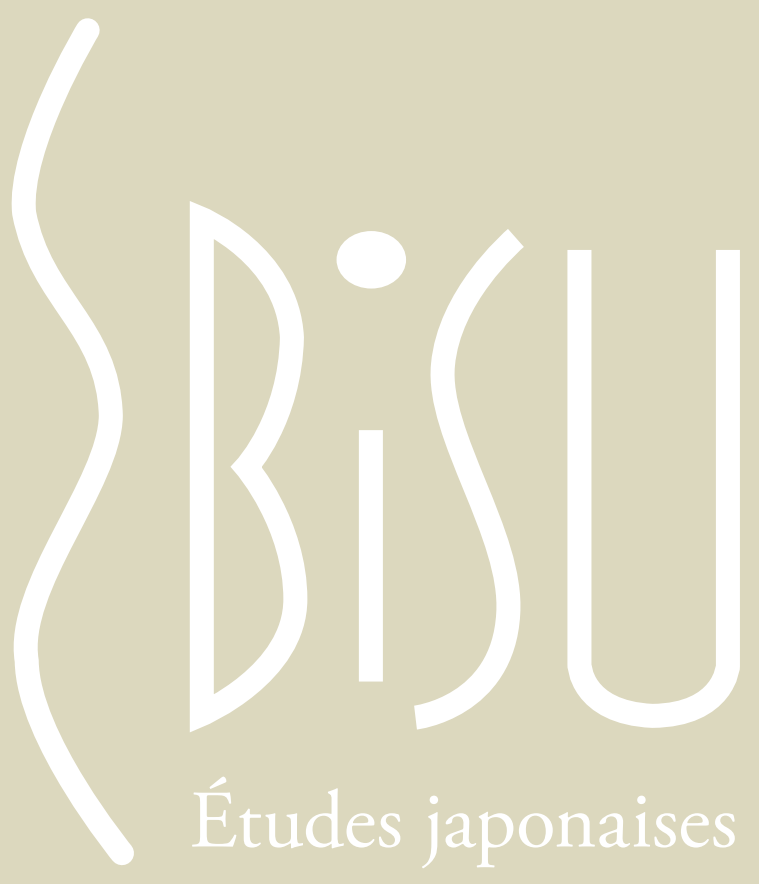

Dossier

De chose en fait : la question du milieu

Articles issus du colloque de Shin-Hirayu

Coordonné par Augustin BERQue

Varia | Nicolas Baumert - Peut-il exister des terroirs du saké ?

Conférences de la MFJ | François Macé - Le Kojiki, une Énéide longtemps oubliée ? | Patrick Beillevaire - Présences françaises à Okinawa : de Forcade (1844-1846) à Haguenauer (1930)

Livres à lire

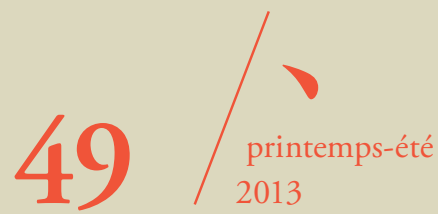




\title{
Peut-il exister des terroirs du saké ?
}

\author{
Nicolas BAUMERT*
}

En tant que boisson de civilisation, qui renvoie à un territoire et à des paysages, le saké est volontiers décrit comme le vin du Japon. Cette analogie peut même être une des traductions du mot nihonshu 日本酒 (Baumert 2006 : 98), et un regard extérieur, en particulier français, voit aisément se dessiner derrière les bouteilles et les savantes calligraphies des étiquettes, des terroirs de rizières ou de montagnes enneigées. La région de Niigata, par exemple, en fait un véritable produit de marketing en insistant sur la qualité de son eau, et les exportateurs usent souvent de cette image exotique pour leur promotion à l'étranger ${ }^{1}$.

Mais existe-t-il vraiment des terroirs du saké ? La réponse n'a rien d'évident, car le mot terroir - terme à l'origine des législations sur la qualité des produits agricoles en France et qui présuppose une relation entre origine géographique et qualité - est loin de s'appliquer naturellement au saké. Tout d'abord, le concept, qui n'a pas de traduction directe en japonais, est plutôt ambigu en français et ses définitions divergent selon les auteurs comme nous le verrons. Ensuite, contrairement au vin qui a été un des

* Université de Nagoya, chercheur associé à l'UMIFRE 19 MAEE-CNRS MFJ.

1. Un exemple parmi d'autres est le site internet www.niigatasake.com qui indique à propos de la qualité de l'eau : "The reason that the Prefecture of Niigata produces the finest sake in the world can be summed up in one word: snow ». Site consulté le 7 juillet 2012. 
premiers produits à bénéficier en France d'une législation fondée sur ce présupposé2, le saké est une boisson qui fait intervenir nombre d'ingrédients dont le principal, le riz, est aisément conservable et transportable. Enfin, la législation japonaise actuelle n'accorde pas véritablement d'importance à l'origine géographique des ingrédients du saké. La question pourrait donc être close. Pourtant, avec la crise des ventes et l'apparition de nouvelles régions de production, loin des traditionnels centres de production du Kansai, le débat s'engage avec pour but de séduire à nouveau, d'une part, les consommateurs japonais, exigeants et souvent blasés par l'abondance de produits de qualité disponibles, mais aussi, d'autre part, les consommateurs étrangers.

La problématique d'une définition de terroirs du saké se place ainsi dans celle, plus large, d'un retour des consommateurs des pays développés à des produits d'origine, qui se manifestait déjà au Japon depuis la fin des années 1990 et qui, dans un contexte post 11 mars 2011, prend soudainement une dimension de sécurité et de traçabilité. Pour bien cerner les enjeux de la mise en place de terroirs dans le contexte japonais, nous proposons une étude géohistorique du saké, partant de la mise en place des premiers territoires de production jusqu'aux expériences actuelles. Le saké est une boisson géographique encore mal définie, dont les critères de qualité sont aujourd'hui remis en question par de nouvelles demandes des consommateurs, ce qui permet de s'interroger sur la pertinence de la notion de terroir appliquée à cette boisson.

2. En France, la réglementation sur les signes d'origine repose sur plusieurs législations qui s'étendent de 1905 à 1935 . Le décret-loi du 31 juillet 1935 portant sur la reconnaissance des appellations d'origine peut être considéré comme l'aboutissement de ce processus qui connaît cependant toujours des modifications, notamment en relation avec l'application des directives européennes sur la protection des indications géographiques. 


\section{Le saké, une boisson géographique encore mal définie}

\section{Une localisation des régions productives marquée par les possibilités de transport}

Ce que l'on entend en français par saké est une boisson alcoolique japonaise issue de la fermentation du riz, dont le riz, l'eau, la moisissure aspergilus oryzae ( $k o ̄ j i$ 粪 en japonais) et les levures sont les ingrédients principaux. Il est fabriqué à l'issue d'un processus de fermentations multiples et parallèles effectué dans des cuves ouvertes, et sa teneur en alcool varie généralement entre $12^{\circ}$ et $17^{\circ}$. Le saké appartient à la catégorie des "vins " de riz, des boissons dont l'aire de diffusion est principalement l'Asie orientale et qui sont produites grâce à un mélange, fait à partir de kōji et de riz, qui permet de convertir l'amidon du riz en sucre, avant de réaliser la fermentation alcoolique grâce à l'action de levures. L'origine de la technique se trouve en Chine dans le bassin du Yangzi et remonte au moins à la période Han (221 av. J.-C.-207 ap. J.-C.) (Huang 2000). Larrivée du vin de riz au Japon n'est pas clairement datée, mais l'hypothèse la plus probable est que la technique chinoise du ferment de kōji ait été apportée via la Corée au v viècle et que cette boisson raffinée ait remplacé un vin de riz plus rudimentaire qui aurait été apporté environ un millénaire plus tôt avec la riziculture ${ }^{3}$.

Dès l'origine, le saké a été dans l'archipel japonais la boisson des dieux et des fêtes. En tant que boisson issue du mariage du riz et de l'eau, sa symbolique a été intégrée dans les divers cultes locaux du shintō ancien et dans les rituels impériaux (Baumert 2008). Dans l'Antiquité, un Bureau du saké existait dans la complexe administration de la cour de Heian et des descriptions dans les chroniques locales (Fudoki 風土記) attestent aussi de sa consommation dans les villages lors des fêtes. Un début de consommation de plaisir s'est également développée chez les nobles autour de la capitale ${ }^{4}$,

3. Il y a probablement eu une boisson issue du riz mastiqué appelée kuchikami no sake 口噛みの酒, qui remonterait à l'introduction de la riziculture au Japon. Pour un point complet sur les différentes hypothèses de l'arrivée du saké au Japon, voir Baumert (2011: 122-130).

4. Il est bien sûr impossible pour cette époque d'avoir une estimation de la consommation, mais, pour prendre un exemple, dans les notes journalières de Fujiwara no Michinaga 藤原道長, ministre à la cour de 995 à 1018, il est fait mention 44 fois de scènes de beuveries ou d'ivresse (Hérail 1987-1991). 
mais il a fallu attendre le Moyen Âge pour qu'un véritable marché se mette en place et que la fabrication du saké devienne une activité commerciale.

Le début du commerce est à relier à des améliorations techniques réalisées dans les monastères de Nara pendant la période Muromachi (1336-1573) (Kamatani 1995). Une meilleure maitrise du processus de fermentation, qui se codifie à cette période, l'utilisation d'un filtrage secondaire du mélange dans des linges de soie, et l'invention d'un processus proche de la pasteurisation sont à l'origine du bond en avant qualitatif d'un nouveau saké appelé morohaku 諸白 (Yoshida 1997 : 16-36). Â partir de ce momentlà, la conservation du produit, donc son transport sur de longues distances, fut possible et des spécialisations régionales sont apparues ${ }^{5}$.

La géographie des régions productrices de saké a été fortement marquée par cette période de la fin du Moyen Âge et du début d'Edo (1603-1867). S’il existait des fabricants dans l'ensemble des fiefs, une région, celle de l'ancien Kinai 畿内, située entre Kyoto, Nara et la baie d'Osaka a longtemps eu le monopole de la qualité. La présence de la capitale impériale qui concentrait la majorité des consommateurs éclairés, prêts à payer cher un bon saké, explique la spécialisation d'artisans dans le saké de qualité au moment où le vin de riz se consommait seulement à proximité de son lieu de fabrication. Les possibilités de transport et la création de la ville d'Edo ne vont pas modifier cette donne, même avec le développement d'une nouvelle capitale loin à l'Est. Malgré la présence de fabriques dans le Kantō, le commerce du saké vers Edo est devenu une source de revenus très importante pour les sakaya 酒屋 (maisons de saké) du Kinai, grâce à la mise en place d'un réseau de transport maritime qui permet un commerce régulier et efficace. Dans ce territoire du saké de qualité, la localisation des productions s'est ainsi rapprochée de la côte, d'abord à Itami 伊丹 et à Ikeda 池田 (près d'Osaka), puis et à Nishinomiya 西宮 et à Nada 灘 (près de Kobe) ${ }^{6}$.

5. Par les choix techniques effectués, en particulier pour le kōji, c'est à ce moment-là que le saké se distingue de ses cousins chinois ou coréens pour véritablement devenir le vin de riz japonais. Les différentes étapes de la fabrication se complexifient et des artisans spécialisés, les tōji $i$ 杜氏, apparaissent. Aujourd'hui encore, malgré la modernisation de la fabrication, les tōji occupent une place centrale dans les fabriques.

6. Yunoki Manabu 柚木学 a publié les chiffres de production et d'exportation de saké des différents sites du Kinai vers Edo pour les années 1784-1786, 1788-1790, 1803, $1805,1817,1821,1837,1843,1853,1856$ et 1866 (Yunoki 1987) ce qui permet d'en 


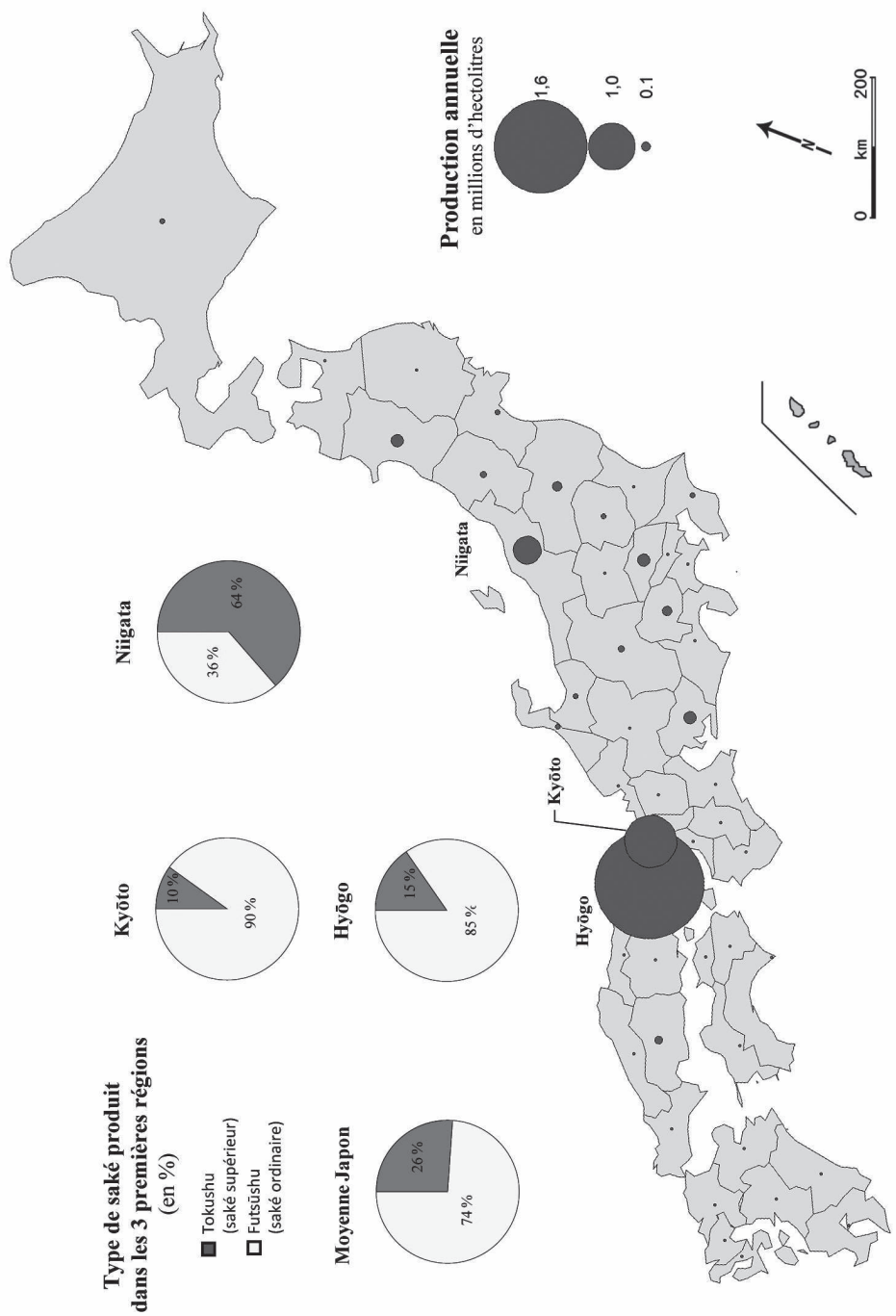

Fig. 1. Production régionale de saké en 2008

Données : Kokuzeichō tōkei nenpōsho 国税庁統計年報書 (National Tax Agency Annual Statistics Report), 2010. Cartographie : N. Baumert. 
À partir de l'ère Meiji et de la modernisation du pays, lorsque le saké est passé progressivement à un stade industriel (Morris-Suzuki 1995), puis, pendant toute la première partie $\mathrm{du} \mathrm{Xx}^{\mathrm{e}}$ siècle, les grandes firmes de Hyōgo et de Kyoto, parce qu'elles ont eu les moyens d'appliquer les progrès techniques, et aussi parce que les transports modernes leur ont permis d'atteindre l'ensemble du territoire, sont devenues les actrices principales au détriment des brasseurs locaux qui ont vu leur nombre se réduire considérablement (Sakurai 1982). C'est cette répartition qui domine encore aujourd'hui la géographie du saké avec $45 \%$ de la production annuelle répartis sur ces deux départements (fig. 1) ${ }^{7}$.

La région composée de l'est de Hyōgo et de Kyoto est majeure pour comprendre l'histoire du saké. Les localisations à grande échelle des fabriques, qui au cours du temps se sont de plus en plus rapprochées de la mer, montrent l'importance de la géographie des transports de l'époque du kudarizake下り酒, ce saké "descendu " du Kinai vers Edo. À l'échelle du pays, l'inertie de ces territoires au cours du temps rejoint les observations faites sur le vin par Roger Dion à propos des vignobles français, dont la géographie actuelle est aussi largement héritée du Moyen Âge (Dion 2010 : 1-61). La logique de ces éléments est toutefois bouleversée par la période actuelle, où l'ensemble des territoires est rendu accessible et où la technique de production du saké est partout maîtrisée. Comme dans un pays aussi montagneux et aussi arrosé que le Japon, une bonne eau se trouve facilement, et comme le riz est présent en abondance, les lignes de force sont égalisées et, d'une manière assez logique, les facteurs locaux de qualité de l'eau se retrouvent ainsi mis en avant. C'est le cas pour les régions récentes de développement de la production de saké, comme Niigata, mais aussi pour nombre de petits territoires jusque-là inconnus. La modernisation des

observer l'évolution. Pour une analyse cartographique du système d'Edo, voir Baumert (2011: 166).

7. La majorité de la production annuelle est faite par un nombre réduit de fabricants. En 2008, douze brasseurs concentraient plus de la moitié de la production. Ces grandes firmes sont pour la plupart localisées dans les vieilles régions de production, comme Kyoto, Itami et Nada. La première brasserie du classement que l'on pourrait qualifier de " régionale » est Asahi shuzō 朝日酒造, une brasserie de Niigata qui arrive en $15^{\mathrm{e}}$ position. Sa production est plus de huit fois inférieure à celle des premières. 
techniques bouscule ainsi l'héritage de l'époque d'Edo, ouvrant le chemin vers de nouveaux types de délimitation des territoires.

\section{Une qualité définie principalement par des critères techniques}

$\mathrm{Au}$ Japon, la législation du saké est gérée au niveau national par le ministère des Finances. Cela s'explique par l'importance du saké dans les recettes de l'État au début de la mise en place de la taxation sur les alcools, à la fin du XIX ${ }^{e}$ siècle (Aoki 2006). La législation actuelle repose sur deux éléments techniques de fabrication. Le premier concerne les ingrédients, en observant s'il y a ou non un ajout de produits autres que le riz, le kōji, l'eau et les levures dans le mélange de fermentation. Dans ce cas, c'est surtout le pourcentage d'alcool industriel ajouté qui détermine la catégorie (la limite maximum d'alcool ajouté ne peut pas dépasser $25 \%$ ), mais il peut aussi y avoir des ajouts d'acides aminés, de conservateurs ou de rehausseurs de goût dans les catégories les plus basses de vin de riz. D’autre part, la législation concerne le degré de polissage du riz utilisé lors de la fabrication. Les catégories les meilleures sont celles dont le riz a été le plus poli et les catégories

\begin{tabular}{|c|c|c|}
\hline & \multicolumn{2}{|c|}{ Critères } \\
\hline & 1. Ingrédients & 2. Polissage du riz \\
\hline \multirow{2}{*}{ Saké supérieur } & \multirow{2}{*}{$\begin{array}{l}\text { Junmaishu 純米酒 } \\
\text { (riz, eau, kōji } \\
\text { et levures uniquement) }\end{array}$} & $\begin{array}{l}\text { Daiginjō 大吟醸** } \\
\text { (moins de } 50 \text { \%) }\end{array}$ \\
\hline & & $\begin{array}{l}\text { Ginjō 吟醸** } \\
\text { (entre } 50 \text { et } 60 \text { \%) }\end{array}$ \\
\hline \multirow{2}{*}{ Saké ordinaire } & \multirow{2}{*}{$\begin{array}{l}\text { Futsūshu 普通酒 } \\
\text { (additions autorisées, } \\
\text { en particulier d'alcool } \\
\text { jusqu'à } 25 \% \text { du volume } \\
\text { de riz) }\end{array}$} & $\begin{array}{l}\text { Honjōzō 本醸造** } \\
\text { (entre } 60 \text { et } 75 \% \text { ) }\end{array}$ \\
\hline & & $\begin{array}{l}\text { Futsūshu 普通酒 } \\
\text { (plus de } 75 \text { \%) }\end{array}$ \\
\hline
\end{tabular}

Fig. 2. Les catégories du saké selon la classification actuelle

* Les pourcentages indiquent le volume résiduel.

** L'ajout d'alcool n'est autorisé que pour ces catégories, jusqu'à $10 \%$ du volume de riz.

Source : Baumert (2009: 335), d'après Kokuzeichō tōkei nenpōsho. 
inférieures sont celles dont les grains n'ont subi qu'un faible polissage, voire aucun (fig. 2).

Cette classification date du milieu des années 1970, lorsque la baisse de la consommation a commencé à se faire sentir et que les syndicats de producteurs ont éprouvé le besoin de donner à leurs produits plus de lisibilité pour les consommateurs. Elle a remplacé l'ancienne classification en " niveaux» (kȳu 級) qui datait du début de Meiji et qui était simplement fondée sur le degré d'alcool. Ce choix s'expliquait à l'époque par la nature de la fabrication lors de la mise en œuvre de la législation. Lorsque le degré d'alcool ne pouvait être obtenu que de façon naturelle, un saké très alcoolisé révélait une bonne maîtrise du processus des fermentations. Avec l'évolution technique et les ajouts d'alcool, elle avait perdu tout son sens. La première tentative de rééquilibrage date de 1969, avec la création du saké de type honjozzo 本醸造, qui limitait l'ajout d'alcool à $10 \%$ du volume de riz. Les catégories portant sur le polissage datent de 1982. Pendant un temps, les deux systèmes ont coexisté et la classification en kyū a été définitivement abandonnée en $1992^{8}$.

Cette classification hybride et incomplète pose de nombreux problèmes. Tout d'abord, les catégories se superposent sans avoir le même but. L'une part de l'idée qu'un bon saké est nécessairement sans alcool ajouté, l'autre qu'un bon saké est avant tout obtenu avec un riz très poli. Ensuite, cette classification est le résultat de la lutte qui se poursuit encore entre les fabricants et du débat non tranché sur la véritable nature du saké. Est-il seulement un produit issu de la fermentation du riz dans de l'eau, ou est-ce que l'ajout d'alcool est possible ? Lors de la mise en place de la législation, les grandes marques, principales pourvoyeuses de sakés ordinaires, n'ont jamais accepté que les sakés avec de l'alcool ajouté ne soient plus considérés comme des seishu 清酒", d'où le flou actuel concernant les meilleurs produits dans lesquels une petite part d'alcool industriel peut être ajoutée.

8. Pour le junmai 純米, plusieurs évolutions de la législation ont eu lieu depuis les années 1980 afin de donner un minimum de polissage du riz à cette catégorie. La dernière, de 2004, va dans le sens contraire, ce qui rend la correspondance entre les deux échelles de qualité de plus en plus difficile.

9. Littéralement seishu signifie "saké filtré ». Dans les classifications, ce terme est utilisé pour désigner le vin de riz. C'est cette dénomination qui est indiquée sur les bouteilles. Nihonshu, terme d'usage courant, ne correspondant à aucune définition technique. 
L’origine du saké est le grand absent de la législation qui est fondée sur la mise en avant de la marque et du processus de fabrication. Comme la législation oblige seulement à indiquer sur l'étiquette la date et le lieu de mise en bouteille, les ingrédients, ou bien le saké lui-même, peuvent venir d'ailleurs. L'indétermination géographique fut à l'origine des nombreux scandales qui ont marqué les années 1970 et 1980, lorsque la presse a révélé que la plupart des grandes marques de Nada et de Fushimi commercialisaient sous leur nom des coupages venant de tout le pays (Kondō 1994 : 89). Par rapport à la législation, rien n'était illégal, en revanche les consommateurs ont ressenti cela comme une trahison. Aujourd'hui, avec le renversement de l'image des régions, c'est plutôt le contraire qui se passe et bien des sakés industriels sont présentés à tort comme des sakés régionaux. Le hiatus qui existe entre la législation sur le saké et les attentes des consommateurs pose un sérieux problème. Il démontre que les Japonais sont particulièrement sensibles à l'origine, que celle-ci semble naturelle à beaucoup d'entre eux, et que l'indication de la région d'où vient le produit implique une confiance et une sensibilité particulière que les standards de fabrication ne garantissent pas. Cette différence entre la législation et ce que l'on pourrait nommer le " pays réel » est révélatrice d'une crise à laquelle de nouvelles réponses doivent être apportées.

\section{Des critères de qualité remis en question par une demande d'authenticité de la part des consommateurs}

\section{Les changements des consommateurs et l'apparition de nouvelles régions de production}

Entre la fin de la Seconde Guerre mondiale et le début du Xxi siècle, le comportement des consommateurs japonais en termes de consommation de boissons a radicalement changé. En un peu plus d'une cinquantaine d'années, le saké est passé, dans le total de la consommation des boissons alcoolisées, de la boisson la plus consommée (environ $60 \%$ ) à une part avoisinant les $10 \%{ }^{10}$. Cette baisse est intervenue dans un contexte de

10. L'ensemble des chiffres cités provient des statistiques annuelles de la National Tax Agency (Kokuzeichō tōkei nenpōsho). 
hausse de consommation des autres boissons. Au cours de cette période, la

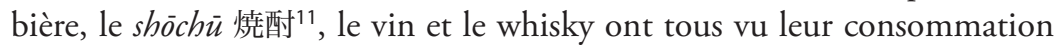
augmenter et cette augmentation s'est principalement faite au détriment du saké. Le saké a perdu sa place de boisson de consommation quotidienne et populaire et a évolué pour les consommateurs vers un statut encore mal défini. Cette évolution affecte des équilibres qui semblaient immuables et modifie en profondeur la géographie de la consommation sur l'archipel où - mis à part les cas particuliers de Kyūshū et de Hokkaidō où la composante culturelle régionale est importante - les différences de consommation qui apparaissent sont plutôt le fait d'un coefficient de ruralité et d'âge de la population (Baumert 2011 : 208-212).

Le changement des habitudes de consommation réside principalement dans l'évolution du Japon au cours de la seconde partie du $\mathrm{xx}^{\mathrm{e}}$ siècle. Pendant cette période, à la manière de tous les consommateurs des pays développés, les consommateurs de saké sont devenus plus exigeants. Ils boivent moins, mais mieux, et ils recherchent la qualité et l'originalité, ce qui se remarque très bien avec l'augmentation de la consommation des sakés supérieurs (ibid. : 200). Surtout, les Japonais n'hésitent plus à consommer d'autres boissons lorsque le saké ne répond plus à leurs attentes. Si les anciennes générations buvaient n’importe quoi du moment que cela s'appelait saké, le consommateur actuel est beaucoup plus difficile, d'autant qu'il a le choix et qu'il a été habitué depuis longtemps aux goûts et aux critères de qualité internationaux.

À cette évolution, les fabricants de saké ont dans l'ensemble mal répondu. Depuis Meiji et l'ère de la consommation de masse, il s'agissait surtout pour eux de satisfaire un besoin quantitatif, et brasser du saké était devenu une affaire industrielle. Dans un contexte qui était celui d'une augmentation régulière de la demande, plus les grandes firmes produisaient et mieux elles distribuaient leurs produits, plus elles gagnaient d'argent. Lorsque la baisse de la consommation de saké a commencé au milieu des années 1970, beaucoup en étaient encore à chercher à accroître les rendements et à faire baisser les coûts de production. La mauvaise image qu'a eue le saké dans

11. Le shōchü est une boisson obtenue par la distillation de diverses bases (riz, blé, pomme de terre, etc.). Elle titre entre 25 et 45 degrés d'alcool selon les types. Historiquement, ce sont des boissons du sud du pays (en particulier de Kyūshū). 
les années 1970-1980 vient surtout de cette période d'après-guerre où la législation tenait peu compte de la qualité et où le niveau moyen de la production était très médiocre.

L'apparition de régions rivales à celle des grands brasseurs de Kyoto et de Hyōgo est révélatrice du retournement des consommateurs. Entre 1983 et 2003, dans une période difficile pour l'ensemble des fabricants de saké, la seule région du Japon qui a vu sa production augmenter de $10 \%$ n'est pas située dans le Kinai mais dans le Nord, sur la mer du Japon. Il s'agit de Niigata. Cette évolution est un renversement des équilibres en place. Pour la première fois depuis le Xvi siècle, et encore plus depuis Meiji où s'était amorcée la phase de concentration industrielle du saké, une autre région a pris de l'importance face à l'ancien Kinai.

L'histoire du succès du saké de Niigata est particulièrement révélatrice. Jadis région pauvre et enclavée, ses producteurs de saké ont su les premiers faire le pas vers la qualité au début des années 1980. Leur réussite tient en trois points : un saké sec qui correspondait à l'évolution du goût des consommateurs, un degré de polissage du riz très important, gage de qualité et, enfin, l'image de pays froid et de neige qui caractérise la région, car l'hiver était la saison traditionnelle du saké12. Preuve de l'apparition d'un nouveau territoire, Niigata est aujourd'hui la seconde région productrice de riz à saké derrière Hyōgo. Elle est aussi la région où la part du saké supérieur dans la production totale est la plus importante (voir fig. 1). Le succès se poursuit depuis trente ans et s'étend aujourd'hui aux départements voisins comme Ishikawa, Akita et Yamagata.

Le renversement de l'image du jizake 地酒, ce « saké de pays » que l'on traitait avec mépris et qui est devenu un "saké de terroir », est à la base de l'évolution en cours. Il permet un retour des régions. Paradoxalement, ce sont donc les brasseries qui avaient raté la première phase de la modernisation, dans des régions autrefois enclavées, où le saké n'était destiné qu'à une consommation locale, qui émergent. Elles profitent d'une image de qualité et de maintien d'une tradition ancestrale désormais mise en valeur. Cette inversion fonctionne car les valeurs associées au saké et les représentations

12. Avant l'introduction des techniques modernes de contrôle des températures, les bons sakés étaient toujours brassés en hiver car le froid permettait de mieux contrôler les fermentations. 
des consommateurs ont évoluée. En effet, les sakés des régions du Nord correspondent à un saké rural, dont la qualité repose sur les critères de pureté de l'eau et sur ses variétés de riz, contrairement au saké des anciennes régions du kudarizake, aujourd'hui pratiquement toutes incluses dans la conurbation Osaka-Kobe-Kyoto, qui représentent certes un saké de vieilles maisons, mais qui est perçu comme un saké industriel et urbain. Dès lors, pour les nouvelles régions, une protection de l'origine par des labels ou des systèmes d'indications géographiques devient indispensable pour donner aux consommateurs les moyens d'effectuer leurs choix, comme le montrent plusieurs expériences en cours depuis 1997.

\section{Les expériences en cours concernant la mention de l'origine}

Niigata a été la première région à proposer, par l'intermédiaire de son association des brasseurs, un label d'origine sur ses bouteilles, en s'inspirant directement de la législation française des appellations d'origine contrôlée (AOC), puisque le nom choisi a été « Niigata-o-c ». Ce label, créé en 1997, combine des critères de lieu de production, d'origine des ingrédients, de technique de fabrication et de qualité minimale du produit. Il est représenté par cinq grains de riz figurant une fleur sur fond bleu, symbole apposé au dos des bouteilles de saké. Pour bénéficier du label, le fabricant doit présenter un produit respectant plusieurs caractéristiques : utiliser une source d'eau du département (donc y avoir sa brasserie), utiliser un riz produit dans le département et le polir au moins à hauteur de $60 \%$ et, enfin, recevoir l'approbation d'une commission de contrôle de l'association des brasseurs $^{13}$. Aujourd'hui, plus de la moitié des fabricants du département (48 sur 92) possèdent ce label de qualité qui en fait le plus important du Japon.

Après Niigata, et certainement en raison du succès rencontré, le département de Nagano a développé en 2002 un système similaire, où le riz utilisé pour le brassage doit être produit dans le département et poli au moins à hauteur de $70 \%$. En 2004, c'est le département de Saga qui a créé son label d'origine, tandis que cinq fabricants d'Ishikawa ont mis en place une appellation centrée sur la région montagneuse de Hakusan 白山 (fig. 3).

13. Voir : www.niigata-sake.or.jp/torikumi/chisan/index.html (consulté en juin 2012). 

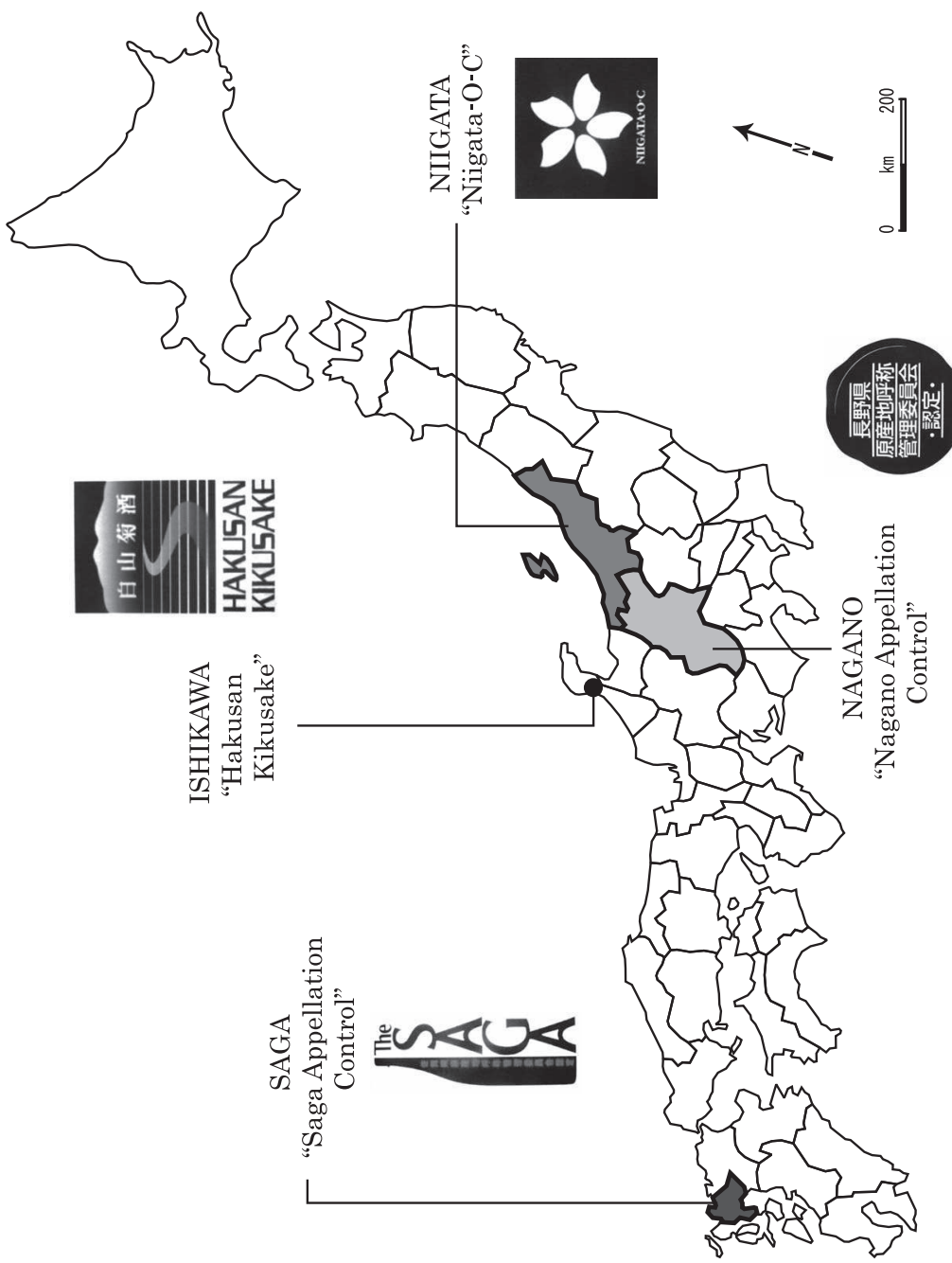

Fig. 3. Les appellations d'origine en 2012

Cartographie : N. Baumert 
L'appellation d'origine déposée du département d'Ishikawa, appelée "Hakusan Kikusake " 白山菊酒 est la seule qui soit gérée à l'échelon national depuis 2005. L'histoire de ce qui est en réalité un trademark associant plusieurs fabricants de la région est significative des enjeux de l'appellation. Ce qui a motivé cette expérience est la réutilisation du nom Hakusan pour d'autres vins de riz, y compris hors du Japon. En 2004, un label a d'abord été créé avec les conditions suivantes : utilisation d'un riz à saké (pas obligatoirement cultivé dans la région) polis au minimum à $70 \%$, obligation de réaliser toutes les étapes du brassage dans la limite de la région de Hakusan et d'utiliser l'eau du bassin du fleuve Tedorigawa 手取川, utilisation d'un minimum de $20 \%$ de riz-kōji dans le mélange et utilisation exclusive des méthodes traditionnelles pour le moto $\overrightarrow{\text { 元 }}^{14}$. Les producteurs doivent ensuite répondre aux exigences d'une commission de contrôle de qualité. Une fois le label en place, le ministère des Finances japonais a ensuite accepté le nom Hakusan Kikusake dont l'utilisation est désormais protégée et limitée aux cinq fabricants du label ${ }^{15}$.

La référence au système français des appellations d'origine est visible à travers les différentes expériences en cours au Japon, Elle se remarque dans les noms donnés, «Appellation control » ou « Niigata-o-c », mais aussi dans le rapport officiel du Kokuzeichō (National Tax Agency) sur l'expérience d'Ishikawa où la législation française des Appellations d'origine contrôlée est mentionnée comme un modèle qui pourrait être suivi en cas de succès ${ }^{16}$. Cette référence aux $\mathrm{AOC}$ et aux terroirs intervient justement à un moment où en son sein même, en France, ce système est de plus en plus controversé et remis en question par la rente territoriale qu'il implique (Hinnewinkel 2002 : 249-252). Pour les petits producteurs de saké dont les régions ont acquis une certaine reconnaissance, il apparaît néanmoins comme un idéal qui permettrait de redonner de la crédibilité à leurs produits, associant l'origine à la qualité.

14. Le moto est un ferment très dense composé de riz contaminé par le kōji et de levures. Il sert de starter à la fermentation du saké.

15. Voir : www.hakusan-kikusake.jp (consulté en juin 2012).

16. Voir : www.nta.go.jp/shiraberu/senmonjoho/sake/hyoji/chiri/kokuji941228/02.html (consulté en juin 2012). 


\section{Le terroir, une notion pertinente pour le saké ?}

\section{L'ancienneté d'une association entre l'origine et la qualité}

La question de l'origine des produits et de la qualité qui lui est associée est au Japon un phénomène ancien. Sans forcément remonter aux indications de crus figurant dans le Man.yōshū 万葉集 ou dans les Contes d'Ise, le saké a fait partie à l'époque d'Edo des premiers produits qui indiquèrent leur lieu de production. Dès le XVII ${ }^{\mathrm{e}}$ siècle, lors du grand commerce entre la région du Kinai et la capitale shôgunale, les sakés d'Ikeda et de Nada mettaient la marque de leur région sur leurs tonneaux et, déjà à cette époque, des contrefaçons existaient, entraînant des plaintes (Yoshida 1997 : 35-47). Cet exemple montre qu'autrefois le nom du fabricant et l'origine du saké étaient intrinsèquement liés dans l'appréciation de la qualité et de la valeur des sakés. Finalement, ce n'est qu'avec l'industrialisation d'une partie du monde du saké que la marque et les critères techniques se sont imposés, entre la fin du XIX ${ }^{\mathrm{e}}$ siècle et le retournement des valeurs associées aux régions dans le dernier tiers du $\mathrm{xx}^{\mathrm{e}}$ siècle.

Le Japon est un vieux pays agricole qui a, au moins dans sa mythologie officielle, construit sa civilisation sur une mise en valeur du territoire par la riziculture irriguée (Ohnuki-Thierney 1993) ${ }^{17}$. Liidée de goût géographique y est bien ancrée dans la culture alimentaire et, pour le saké, ce phénomène se retrouve dans l'ancienneté de la relation entre les traditions de fabrications et la cuisine de chaque région. En reprenant la séparation classique entre l'Est et l'Ouest du pays ${ }^{18}$, il s'avère effectivement qu'entre les deux Japon le saké se fait différemment, en s'adaptant à la cuisine. Globalement,

17. Il s'agit d'une mythologie officielle, car, à l'époque Heian, le riz n'était pas forcément la seule céréale, et son importance dans l'imaginaire collectif était concurrencée par l'orge, le blé et le millet qui faisaient aussi partie des offrandes aux divinités (Von Verschuer 2003). La place exclusive du riz n’intervient que plus tard, à la fin du Moyen Âge.

18. L'alimentation de l'Ouest du Japon se distingue par les grosses nouilles de blé (udon) et des assaisonnements légers préparés à base d'algues, tandis qu'à l'Est prédominent les fines nouilles de sarrasin (soba) et des assaisonnements plutôt salés au goût de shōyu très prononcé. Okinawa et la partie méridionale de Kyūshū se distinguent quant à elles par leur influence chinoise avec une cuisine plus grasse et une consommation importante de porc. La boisson alcoolique traditionnelle est le shōchī, un alcool distillé. 
il est plus sec et frais à l'Est, tandis qu'à l'Ouest, il est doux et rond. Les sakés de Kyoto sont plutôt faits pour accompagner les mets et ceux du Tōhoku sont plus pris comme éléments centraux du repas, avec des accompagnements aux goûts très relevés ${ }^{19}$.

L'ancienneté de l'association entre origine et la qualité se retrouve aussi dans la langue japonaise, où trois termes sont souvent appliqués aux ingrédients ou au savoir-faire des productions agroalimentaires : nōsanchi 農産地 (propriétés agronomiques du sol), chihō 地方 (territoire) et kyōdo 郷土 (identité du lieu). Il est aussi possible d'associer le concept de füdo 風土 (milieu/médiance ${ }^{20}$ ) dans le sens donné par Watsuji Tetsurō 和辻哲郎 (Watsuji 2011), un concept qui à l'origine a été conçu pour expliquer les relations homme-milieu, mais qui peut également s'appliquer à la nourriture conçue comme lien organique entre l'homme et son environnement. Dans cette optique, le milieu fournit à la fois les ingrédients de la cuisine, mais leurs productions et les spécialisations qu'elles demandent modifient aussi, par des choix humains, les paysages (Fukuda 2011). Depuis les années 2000, cette attention au territoire s'exprime particulièrement dans le goût pour la consommation des produits de terroir, dans les paysages même qui les ont vus naître. Cette mode, caractérisée par la formule chisan chishō 地産 地消 que l'on peut traduire par "produire et consommer au pays ", s'apparente à un art de vivre et permet le développement de production de qualité pour le riz, la viande ou les légumes, et l'apparition de circuits de distribution courts et de commercialisation directe (Shimohirao et al. 2009).

Toutefois, malgré ce contexte, pour beaucoup de décideurs, le saké est longtemps resté une boisson qui devait faire référence à la marque et non au lieu de production, ce qui explique l'orientation de la législation actuelle. Les raisons en général invoquées pour ne pas ouvrir le débat sur l'origine étaient la multiplicité des ingrédients du saké et l'absence du concept de terroir dans la langue japonaise. Cette argumentation est partisane et peu convaincante. En effet, même si le mot « terroir » est souvent simplement

19. La différence entre boire et manger passe au Japon principalement par les éléments principaux issus du riz consommés (gohan, saké, mochi) qui sont rarement présentés ensemble. "Boire le saké » signifie donc prendre, en quelque sorte, un repas sans riz solide, mais avec des accompagnements spécifiques (Cobbi 1999).

20. Pour une explication du concept et sa traduction en français par médiance, voir la préface d'Augustin Berque à l'ouvrage de Watsuji (2011 : 11-28). 
transcrit phonétiquement (terowāru テロワール), la langue japonaise possède l'ensemble des notions qui s'y rapportent. D'autre part, il existe une véritable demande des consommateurs de prise en compte de l'origine, contre les abus permis par la législation actuelle. Une des explications de cet écart est le fait que, de par son histoire, le lien entre le monde du saké et la politique est extrêmement fort. De la figure du brasseur de saké dans les villages au prêteur d'argent des grandes sakaya de l'époque Muromachi (Gay 2001), en passant par la modernisation du pays sous Meiji et la mise en place de la taxation sur les alcools (Hurt 2000), les fabricants de saké ont toujours été proches du pouvoir et du monde de la finance, à tous les niveaux de la société. C'est peut-être ce qui explique encore aujourd'hui la législation qui favorise les grandes marques, alors qu'un autre produit traditionnel, le shōchü, s'est doté de critères de qualité liés à l'origine pour protéger les régions historiques du sud du pays, lorsque cette boisson est devenue à la mode dans les années 1990. La législation sur le shōchū est aujourd'hui claire et gérée au niveau national, alors que ses ingrédients sont bien plus divers que ceux du saké ${ }^{21}$.

\section{Quels terroirs pour quels sakés?}

Sur l'idée d'une protection prenant en compte l'origine géographique, les avis entre les partisans et les opposants sont extrêmement tranchés, mais semblent en évolution. Dans les résultats d'une enquête réalisée en 2009 auprès de 121 producteurs de saké (Baumert 2009 : 402-407), il est apparu une demande de prise en compte de l'origine par $60 \%$ des sondés. Parmi les 73 fabricants favorables à une prise en compte plus importante de l'origine, 32 réponses allaient dans le sens d'une législation inspirée des terroirs à la française et 41 considéraient que le saké était trop différent pour pouvoir être envisagé de cette manière, indiquant toutefois qu'il faudrait trouver une solution différente, mieux adaptée au saké. Quatre brasseurs indiquaient par ailleurs qu'eux-mêmes produisaient un saké que l'on pourrait qualifier «de terroir », prenant en charge sur place la totalité des étapes de la fabrication du saké, de la culture du riz jusqu’à la mise en

21. Voir : www.nta.go.jp/shiraberu/senmonjoho/sake/shiori-gaikyo/2012/pdf/009.pdf, p. 53-56 (consulté en juin 2012). 
bouteille (Baumert : 344). Ces résultats reflètent à la fois des oppositions entre cultures régionales, taille des fabricants, mais aussi, plus fondamentalement, des différences de conception quant à la véritable nature du saké.

Paradoxalement, pour une boisson considérée comme identitaire à l'étranger, au Japon, la question qui pose problème est : qu'est-ce que le saké ? Les problèmes de dénomination et de définition du saké, qui s'expriment dans l'écart entre l'appellation courante nihonshu et le terme seishu indiqué sur les étiquettes, ne posent pas que des interrogations purement théoriques. Les professionnels tournent pour le moment autour du sujet sans véritablement y apporter de réponse, car, ce qui fait débat, c'est la nature du saké depuis les années de modernisation qui ont, grâce aux avancées techniques, permis divers ajouts. Le saké est-il seulement le produit d'une fermentation de riz dans de l'eau pure, ou bien peut-il y avoir différents ajouts ? La législation avec ses deux échelles ne tranche pas et laisse les interprétations ouvertes.

Pareillement, le concept de terroir présente une multiplicité d'acceptions et de définitions. Elles vont d'une définition simpliste impliquant une relation quasi déterministe entre le goût du produit et son origine, à une définition complexe intégrant les différentes composantes humaines et culturelles. Ainsi, d'après la définition proposée par un groupe de travail INRA/INAO et présentée à l'UNESCO en 2005, le terroir est " un espace géographique délimité où une communauté humaine a construit, au cours de l'histoire, un savoir intellectuel collectif de production fondé sur un système d'interactions entre un milieu physique et biologique et un ensemble de facteurs humains" (Casabianca et al. 2008). En reprenant les travaux d'Emmanuelle Vaudour, il est possible de distinguer différentes facettes du concept de terroir, qui font intervenir quatre aspects : le "terroir-matière " qui pose la relation avec le milieu physique, le "terroir-espace » qui le lie à un découpage spécifique, le "terroir-identitaire " qui donne à l'espace une profondeur historique et culturelle et le " terroir-slogan " qui reprend ces éléments comme argument de vente en les simplifiant et en créant, par là même, des représentations nouvelles (Vaudour 2003 : 9-13).

Avec une définition large du terroir, considérant le saké comme une boisson alcoolique issue de la fermentation du riz et dont le riz, l'eau, la moisissure kōji et les levures sont les seuls ingrédients, l'élaboration de terroirs du saké semble donc possible. Au regard des éléments historiques et géographiques, les caractéristiques du saké reprennent l'ensemble des dimensions du concept de terroir : le riz et l'eau définissent le "terroir-matière ", 
la profondeur historique des territoires créant de nombreux «terroirsidentitaires » et les nouvelles régions utilisant le " terroir-slogan " pour la promotion de leurs produits. Le seul élément qui manque, malgré les tentatives de création de labels et de trademarks associant l'origine, est le " terroir-espace " qui les lierait à un découpage spécifique géré au niveau national. Si, en raison de l'importance dans l'histoire de la boisson, des marques et du négoce, une législation uniquement basée sur l'origine ne peut pas, en l'état actuel des choses, être érigée en but ultime et représenter l'ensemble de la gamme des sakés, le retour à une association entre la marque du fabricant, le lieu de production et l'origine des ingrédients permettrait néanmoins de protéger ceux qui tentent de faire du saké un produit original et représentatif des paysages et des hommes qui le produisent.

\section{Vers des jizake de terroir}

Depuis le retour du jizake et l'apparition de nouvelles régions, le terroir est devenu une image et un slogan. Mais, de la même manière que pour bien d'autres productions, ce terroir, qui est perçu comme réceptacle d'une originalité, est pour les consommateurs comme le paysage : il est «dans le regard, pas dans l'espace" (Maby 2003 : 274). Tant que la représentation fonctionne, le lien posé entre la qualité et le lieu de production est accepté, toutefois, dès lors que la chaîne est rompue, le lien se trouve remis en question. C'est ce qui s'est passé avec la modernisation du monde du saké à partir de Meiji, quand les critères industriels de fabrication ont pris le pas sur l'origine des sakés, puis lorsque les problèmes non résolus sur la qualité ont fait revenir le débat sur l'origine. Les régions du Nord ont alors recréé l'histoire de leurs sakés à partir de critères physiques, comme la qualité de l'eau liée aux fortes précipitations neigeuses, ou la qualité du riz.

Pour le jizake, les critères physiques concernant le lieu de fabrication de la boisson et de ses ingrédients introduisent en effet une relation verticale liée au sol et au climat, qui sont mis en avant par les labels et permettent aussi d'identifier des paysages spécifiques. Parmi les quatre ingrédients du saké (eau, riz, levures, kōji), l'eau est l'élément le plus déterminant, car elle ne peut pas être déplacée. Dans la localisation de la brasserie, la source d'eau est donc primordiale et sa qualité fait intervenir des différences ponctuelles, que l'on peut qualifier de géographiques, dans les goûts des sakés. Le riz est un second aspect de la question. L'introduction de variétés nouvelles 
adaptées au climat de chaque région, fruit de la recherche agronomique qui s'est développée depuis les années 1920, donne une identité des riz qui résulte à la fois de choix humains et de contraintes physiques. Le riz n'est donc plus uniquement une matière première quelconque, mais un élément territorialisé et les nouvelles variétés, avec leurs spécificités régionales, peuvent donc être, toutes proportions gardées, assimilées aux cépages du raisin, entraînant également une différenciation au niveau du goût.

En raison de l'histoire de la fabrication du saké et des savoirs-faire mis en œuvre, les délimitations uniquement physiques, basées sur le lieu de production des ingrédients, ne peuvent néanmoins être totalement valables. Le saké est une boisson faisant intervenir plusieurs ingrédients, qui peuvent être conservés et transportés. Les territoires actuels, nous l'avons vu, se sont mis en place selon d'autres logiques, de transport notamment. De plus, la distinction entre les fabricants de saké et les producteurs de riz correspond à une manière ancienne d'appréhender la boisson. La nature du saké, le nombre d'ingrédients et les combinaisons possibles laissent donc aux choix humains une importance plus grande, qu'il faut prendre en compte pour rendre à d'éventuels découpages toute la complexité des territoires.

En observant les expériences en cours, la prédominance de l'échelle départementale est une caractéristique majeure. Mis à part Ishikawa, tous les autres labels géographiques ont pris pour échelle leur département. Ce choix s'explique par l'organisation du système des associations de producteurs de saké, qui sont justement organisées selon ce découpage et, dans tous les cas, c'est bien de ces associations que sont parties les tentatives. L'importance de l'institution est donc centrale, bien plus que celle d'un éventuel génie des lieux ou du sol. L'absence pour le moment, dans ces expériences sur l'origine des territoires, des plus fameux du kudarizake comme Itami, Nada, Nishinomiya, Ikeda ou Fushimi, trouve aussi ici son explication. Les régions qui ont développé des processus de protection sont celles où la reconnaissance du saké est bien plus récente et qui ont fondé leur qualité sur des critères liés aux ingrédients. Ce sont aussi des régions où dominent les petits producteurs. Par contre, les régions de l'ancien Kinai sont dominées par des grandes marques de saké, déjà reconnues, pour qui une focalisation sur l'origine géographique comme critère de qualité aurait pour conséquence de valoriser d'autres producteurs du même espace et de niveler leurs avantages. 
Les enjeux ont toutefois complètement changé d'échelle. La baisse de la consommation atteint un niveau tel que l'on peut raisonnablement se poser la question du maintien du saké comme produit de consommation courante, tandis que les exportations restent faibles. Sur ces deux points, le ministère de l'Agriculture pourrait d'ailleurs prendre le relais du ministère des Finances pour la réorganisation de la gestion du saké. Le rapport du Bureau national de la prospective (Kokka senryakukyoku 国家戦略局) de 2012 sur l'état du monde du saké l'y engage et parle même de "tragédie " et d' « échec national ». Pour relancer la boisson, il prône une promotion complémentaire des sakés et des shōchū sous l'appellation kokushu 国酒 (boissons nationales) ${ }^{22}$. Autant de signes qui montrent que le passage par une reconnaissance de l'origine des produits devient un critère pour la relance de la boisson. À l'intérieur des régions, une réflexion sur le jizake qui ne correspond encore à aucune définition juridique aurait l'avantage de ne pas modifier les équilibres actuels. Selon les territoires, les terroirs et les conditions justifiant une originalité pourraient être différents, adoptant des découpages appropriés. Si, dans la plupart des cas, c'est l'échelle du département qui apparaît la plus parlante au consommateur, pour les anciennes provinces du Kinai, les découpages pourraient se faire à une échelle plus précise, car leurs territoires sont suffisamment reconnus dans l'ensemble du pays pour faire valoir leur origine sans forcément faire du tort aux marques les plus connues. Un exemple significatif de l'évolution des pratiques est celui du saké Onikoroshi 鬼ころし d'un des grands fabricants de Nada, Kinshika 金鹿, un saké que l'on pourrait qualifier d'industriel, vendu en packs de carton, mais qui indique sur ses étiquettes « $100 \%$ Nada no sake » (Saké $100 \%$ de Nada).

22. Voir : http://www.npu.go.jp/policy/policy04/pdf/20120511/kokushu.pdf (consulté en juillet 2012). 


\section{Conclusion Un contexte favorable?}

S’il existe des sakés de terroir, il n'existe pas encore véritablement de terroirs du saké. La boisson dans l'archipel japonais est un produit bien ancré dans les pratiques alimentaires, elle est le produit d'une relation complexe entre l'homme et le milieu, mais la notion de terroir y est toujours en construction, à la fois sur le plan juridique et dans les représentations des consommateurs. Le débat sur la prise en compte ou non de l'origine du saké rappelle étrangement la crise qui a opposé en France, au début du xx $x^{\mathrm{e}}$ siècle, négociants et propriétaires, et qui a vu l'institutionnalisation de la qualité se faire sur une appartenance ou non à une appellation d'origine contrôlée. Avant les différentes lois, et en particulier celle du 6 mai 1919, l'identification de la qualité du vin se faisait à la fois à partir du nom du village et de celui du négociant. Les propriétaires, grâce à leurs réseaux politiques, ont réussi à faire accepter l'idée que la qualité du vin était due au terroir (physique) dont il était issu (Laferté 2006 ; Jacquet 2010). Le saké a connu à la même période une évolution inverse en voyant les grandes marques imposer leur nom et la modernité de leurs processus industriels dans la définition de la qualité. Aujourd'hui, les enjeux de la prise en compte de l'origine pour le saké sont donc exactement les mêmes que ceux de la France du début du $\mathrm{xx}^{\mathrm{e}}$ siècle et, à la concurrence entre les marques qui a marqué la période d'industrialisation, se superposent une concurrence entre les marques et les régions, ainsi qu'une concurrence entre les régions elles-mêmes. Dans ce foisonnement, le contexte de crise actuel est peut-être favorable et devrait permettre une vraie définition du produit, certainement en relation avec l'origine, comme les expériences en cours peuvent le laisser présager. Pour le saké, comme pour bien des produits de qualité, l'après 11 mars 2011 a entraîné tant un besoin de traçabilité pour la sécurité alimentaire, qu'une exacerbation des rapports au territoire ${ }^{23}$. Ces éléments s'ajoutent à la volonté actuelle du Japon de promouvoir ses produits à l'étranger et, face à la peur occasionnée par la contamination radioactive, il cherche à proposer un art

23. Dans l'année qui a suivi le grand tremblement de terre du 11 mars 2011, les ventes de saké des régions les plus touchées et en particulier de la région de Fukushima ont nettement augmenté suite à un réflexe de solidarité nationale de la part des consommateurs. 
de vivre et des paysages plus que de simples produits ${ }^{24}$. Nihonshu, kokushu, jizake, sont autant d'appellations qui font référence à des espaces vécus et appropriés qui devraient, à l'avenir, si l'effort de définition de territoires se poursuit, permettre de rendre compte, tant au niveau national qu'international, des spécificités géographiques du vin de riz japonais.

\section{Bibliographie}

\section{AOKI Takahiro, 2006}

"Le mouvement anti-alcoolique au Japon, de 1880 à 1920, et l'adoption de la législation antialcoolique pour les mineurs », Ebisu, 35 : 73-95.

BAUMERT Nicolas, 2006

«Modes et mondes du boire : vin et saké dans la mondialisation », Ebisu, 35 : 97-109.

\section{BAUMERT Nicolas, 2008}

«Saké et shintō, l'importance du territoire dans la relation entre le vin et le divin au Japon », Géographie et cultures, 63 : 119-132.

\section{BAUMERT Nicolas, 2009}

Le saké, géographie historique et culturelle d'une exception japonaise, thèse de doctorat, sous la direction de JeanRobert Pitte, université Paris-Sorbonne.

24. Entretien réalisé en février 2012 avec des responsables de la promotion du saké à l'étranger. 
BAUMERT Nicolas, 2011

Le saké, une exception japonaise, Rennes, Presses universitaires de Rennes/Tours, Presses universitaires François Rabelais.

\section{CASABIANCA F., SYLVANDER B.,} NOËL Y., BÉRANGER C., COULON J.B. et RONCIN F., 2008

«Terroir et typicité : deux concepts clés des appellations d'origine contrôlée.

Essai de définitions scientifiques et opérationnelles », in Actes du colloque international de restitution des travaux de recherche sur les indications et appellations d'origine géographiques, INAO-INRA, Produits agricoles et alimentaires d'origine : enjeux et acquis scientifiques, Paris, 17-18 novembre 2005, Paris, INRA éditions, pp. 199-213.

\section{COBBI Jane, 1999}

"Le repas japonais », in COBBI Jane \& FLANDRIN Jean-Louis (dir.), Tables d'hier, tables d'ailleurs, Paris, Éditions Odile Jacob, pp. 389-403.

\section{DION Roger, 2010}

Histoire de la vigne et du vin en France des origines au xixe siècle, Paris, CNRS Éditions.

FUKUDA Ikuhiro 福田育弘, 2011 «Inshoku ga tsukuru fūdo, fūdo ga tsukuru inshoku» 飲食がつくる風土、風土 がつくる飲食 (La nourriture du milieu, le milieu de la nourriture : le trajet entre le paysage et le goût), Waseda daigaku gakujutsu kenkyū, jinbun kagaku shakai kagaku-hen 早稲田大学学術研究一人文科 学社会科学編, $60: 327-352$.
GAY Suzanne, 2001

The Moneylenders of Late Medieval Kyoto, Honolulu, University of Hawaii Press.

\section{JACQUET Olivier, 2010}

« Les appellations d'origine et le débat sur la typicité dans la première moitié du $x x^{e}$ siècle : le rôle du syndicalisme viti-vinicole bourguignon et la création des AOC », in HINNEWINKEL JeanClaude (dir.), Faire vivre le terroir, Bordeaux, Presses universitaires de Bordeaux, pp. 117-128.

HÉRAIL Francine, 1987-1991 Notes journalières de Fujiwara no Michinaga: ministre à la Cour de Heian (995-1018), traduction du Midō kanpaku-ki 御堂関白記, 3 vol., Paris, Publications orientalistes de France.

HINNEWINKEL Jean-Claude, 2002

Les Territoires de la vigne et du vin, Bordeaux, Ferret.

HINNEWINKEL Jean-Claude, 2007

«Vignobles et géohistoire », Sud-Ouest Européen, 23 : 5-16.

\section{HUANG Hsing-Tsung, 2000}

Fermentation and food sciences, Vol. 6 : Biology and biological technology, Part 5, in NEEDHAM Joseph (dir.), 1956-2008, Science and civilization in China, 7 vol., Cambridge, Cambridge University Press.

\section{HURT Pascal, 2000}

" L'assemblée des brasseurs de saké ", Cipango, 9 : 93-132. 
KAMATANI Chikayoshi, 1995

«Sake Brewing and Its Records in Edo Japan », Historia Scientiarum,

56 : 117-125.

Kokuzeichō tōkei nenpōsho 国税庁 統計年報書 (National Tax Agency Annual Statistics Report), 1947-

Tokyo, Kokuzeichō 国税庁.

\section{KONDŌ Hiroshi, 1996}

The Book of Sake, Tokyo, London, Kodansha international.

LAFERTÉ Gilles, 2006

La Bourgogne et ses vins, Paris, Belin.

MABY Jacques, 2003

«Modalités de construction d'un savoir scientifique sur les paysages viticoles », Actes du colloque international Paysages de vignes et de vins, Abbaye royale de Fontevraud 2-3-4 juillet 2003, InterLoire-Office international de la vigne et du vin : 273-277.

MORRIS-SUZUKI Tessa, 1995

«The Great Translation: Traditional and Modern Science in Japan's

Industrialization », Historia Scientiarum, 56 : 103-116.

OHNUKI-TIERNEY Emiko, 1993

«Pureté et soi primordial : la nature japonaise », Géographie et cultures, $7: 75-92$.

SAKURAI Hirotoshi 桜井宏年, 1982 Seishugyō no rekishi to sangyō soshiki no $k e n k y u ̄$ 清酒業の歴史と産業組織の研究 (Étude sur l'histoire de la fabrication du saké et sur l'organisation de l'industrie), Tokyo, Chuō kōron jigyō shuppan 中央公論事業出版.

SHIMOHIRAO Isao 下平尾勲, ITŌ Tsunatoshi 伊東維年 et YANAI Masaya 柳井雅也, 2009 Chisan chishō 地産地消 (Produire et consommer au pays), Tokyo, Nippon hyōronsha 日本評論社.

VAUDOUR Emmanuelle, 2003

Les terroirs viticoles, Paris, Dunod.

VON VERSCHUER Charlotte, 2003

Le riz dans la culture de Heian, mythe et réalité, Paris, Collection de France.

WATSUJI Tetsurō, 2011

Fūdo, le milieu humain, commentaire et traduction par Augustin Berque, Paris, CNRS Éditions.

YOSHIDA Hajime 吉田元, 1997

Edo no sake 江戸の酒 (Le saké de l'époque d'Edo), Tokyo, Asahi shinbunsha 朝日新聞社.

YUNOKI Manabu 柚木学, 1987

Sakezukuri no rekishi 酒造りの歴史 (Histoire de la fabrication du saké), Tokyo, Yuzankaku shuppan 雄山閣出版. 